\title{
Asynclitism and Cerebellar Tentorium Tears in Fetuses during Labor
}

\author{
V. V. Vlasyuk* \\ Head of the pathology Department, Hospital no 122, FMBA of Russia, St. Petersburg, Russia
}

Received: December 14, 2015; Accepted: May 16, 2016; Published: May 25, 2016

*Corresponding author: V.V.Vlasyuk, Professor, head of the pathology department, Hospital No.122, FMBA of Russia, St. Petersburg, Russia E-mail: vasily-vlasyuk@yandex.ru

\begin{abstract}
The paper studied the relationship between tears of Cerebellar Tentorium (TC) and asynclitism. It emphasizes the importance of study of the Area Periosteal blood Stagnation (APS) on the skull of the fetus and newborn. Investigated the relationship between the location of APS and tears TC. It explains why tentorial tears occur more frequently in the left half of it. It is shown that asynclitism is a risk factor tears cerebellar tentorium in the fetus during labor. The review presents an original classification tentorial tears. The author argues that changes in the skull can be diagnosed localization wired head point and the degree of asynclitism.
\end{abstract}

Keywords: Tear cerebellar tentorium; Asynclitism; Head insertion; Area of periosteal blood stagnation; Head configuration

\section{Introduction}

In the world literature does not pay enough attention to the study of the Tentorium Cerebelli (TC), which is the key to unlocking the mechanisms of birth trauma. In basic modern international hand books for pediatric pathology, perinatology and pediatric neurology no answer to the question, what is the mechanism tears TC [1-6]. There are only general data about the tears of TC in the application of obstetrical forceps, vacuum extraction of the fetus and compression of the fetal head $[1,6]$. It describes the nature of the tears TC and associated the subdural hemorrhages. In the works are no answers to the questions why there are often one-sided and left-sided tears MN, as it relates to the insertion of the head, how they relate to changes in the skull and the periosteum, biomechanisms labor and asynclitism. The answers to these questions are still not in special studies of the TC $[7,8,9]$ in birth injury $[10,11]$ and in the leading guidelines for pediatric pathology $[2,3,6]$. The answer to these questions was only in our recent studies $[12,13]$ which are not reflected in the English-language literature.

In the process of births with cephalic presentation of the fetus's head is formed not only birth tumor (caput succedaneum), but also the Area (Region) of the Periosteal blood Stagnation (APS). It occurs as a result of violations of the outflow of blood from the periosteum due to compression of the veins in the zone of contact of the fetal head with the walls of the birth canal of the mother Figure 1 and 2. Therefore, in the field of APS encounter point and spotty hemorrhages that long retains after the birth (unlike caput succedaneum). The APS reflects the nature of the insertion head, changes its location when asynclitism. APS is a reflection (imprint) of delivery, which can be used to objectively evaluate the insertion head. APS is clearly visible on the head of the dead fetuses and newborns. Due to the fact that in the field of APS have small hemorrhages and they need time for resorption, they are seen at autopsy of the deceased children even after 10 days of life.

The APS concept was first introduced by the author of this publication in 1987 [14]. It is known that in childbirth in the tissues of the skull can occur caput succedaneum, subaponeurotic hemorrhage and cephalohematoma. This group of traumatic injuries should be attributed and APS. While only a pathologist can diagnose APS, but, in our opinion, it is possible diagnosis of APS from living children using radiological methods. APS - a rounded area expressed stagnant hyperemia periosteum of bones skull roof in combination with petechiae and spotted bleeding. APS has a border around the circumference, which is more pronounced in full-term infant Figure 1. This venous

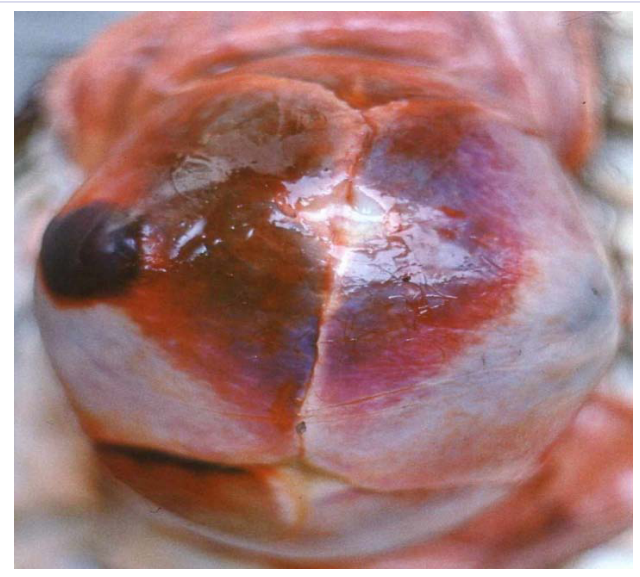

Figure 1: Area of the periosteal blood stagnation on the skull of the dead newborn. The edge area is small cephalohematoma. 


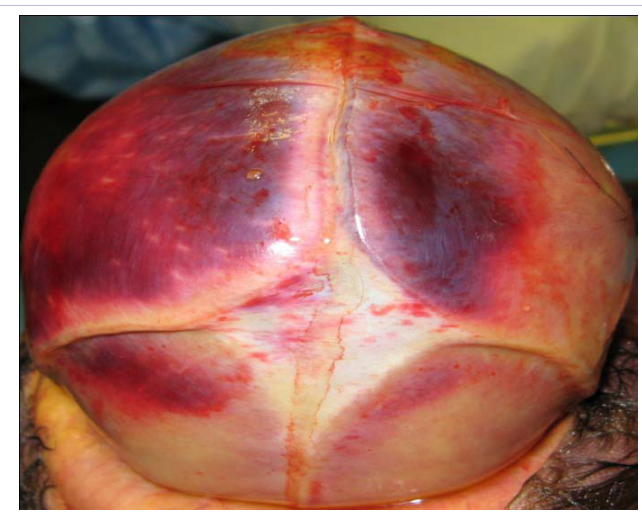

Figure 2: Area of the periosteal blood stagnation, displaced to the right parietal bone on the skull when asynclitism, of the dead newborn, lived 2 days.

congestion with hemorrhages in the periosteum occurs below the zone of contact of the head in the birth canal of the mother. It has no pathological effects on the child, but has important diagnostic value In severe degree of RPC in its place may occur cephalohematoma Figure 1.

From the material of its own 657 unselected perinatal autopsies in 1977-1984 years have been isolated newborns and intrapartum died fetuses who were born with cephalic presentation and who found a clear APS and tears in TC. From this group excluded children born cesarean section, with the presence of externally visible malformations, cases with the use of the obstetric forceps and the vacuum extractor, as well as instances of incorrect presentation of the fetus and abnormal insertion of the head (frontal face). As a result, it investigated 83 dead intrapartum fetal and newborn with tears TC, born with cephalic presentation. All the studied APS of the skull roof and localization of tears TC.

TC is a duplication of the Dura mater, connected with a falx cerebri, which is attached to the bones of the skull. The force of compression of the head and changing its configuration always passed through the falx cerebri on the Tentorium Cerebelli. Because the strength of the Tentorium Cerebelli is almost two times smaller than the falx cerebri, so the TC is damaged in the first place. TC is a "Locus Minoris Resistentiae" birth trauma of the skull.

There are 2 groups of lesions TC:

1) Intradural Hemorrhage and

2) Tears (laceration, rupture).

Tears of TC - the main manifestation of birth trauma to the skull [1,2,6-9]. Tears of TC account for $80 \%-85 \%$ of all fatal injuries [6]. We classify the tears TC as follows. Depending on the side of the lesion tears TC can be 1) unilateral or predominantly unilateral Figure 3 and 2 bilateral, depending on the topography 1) marginal (predominant) and central (at full rupture, it is called perforated), depending on the extent of damage - 1) partial (rupture or tear of one, most of the upper leaf, of the Dura mater) and 2) complete (rupture of two leaves) $[12,13]$. If bleeding in TC not lead to pathological consequences, the tentorial tears can lead to often fatal subdural hemorrhages. In rare cases, TC breaks may extend to the sinuses, especially in the transverse sinus, leading to fatal subdural hemorrhage. However, in the study group were no such cases. When TC tears broken veins, extending between TC and the sheets flow into the sinuses. In the study of APS can accurately determine the location of the head wired point, to accurately measure the degree of asynclitism and accurately characterize insertion of the head [15]. Comparing the location of the APS on the skull and localization of intracranial lesions for the first time were able to identify certain correlations. Earlier pathologists around the world separately described changes of the skull and intracranial lesions, but did not seek their relationships [2,3,7-10]. The correlations between localization of the RPS and the nature of tears CT will be only presented in this paper.

It is found that the displacement of the APS away from sagittal suture to the right Figure 2 or to the left followed by unilateral tears TC $[12,14]$. At the same time the central location APC is accompanied by bilateral tears TC. We have established a pattern (the law Vlasyuk-Lobzin-Nesmeyanov): unilateral rupture of the TC is located on the side opposite to the point shifted the APS $[13,15]$. For example, if an APS is shifted to the right parietal bone, the tears of TC observed in the left half of TC and we should expect a left-sided subdural hemorrhage. If there is a bilateral tear of TC, the tear largest in size is on the side opposite to the direction of displacement of the APS.

It is known that tears TC often one-sided $[16,17]$. On our material in full-term children, born with cephalic presentation, frequency of unilateral rupture towards all the tears TC was $69.2 \%$ [12]. In children born with cephalic presentation in $72.2 \%$ of cases, tears TC one-sided. At the same time, in the group of children with breech presentation tears TC more often bilateral (65.5\%). Some authors believe that the tears TC more often bilateral $[11,18]$, but they are not separated groups of children

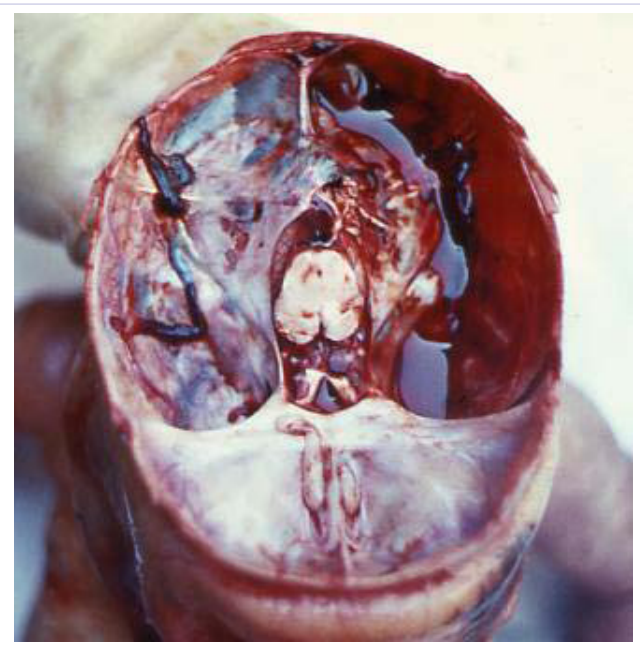

Figure 3: Rupture of the left half cerebellar tentorium in the newborn, lived 2 days 4 hours. Anterior asynclitism (anterior parietal bone presentation). 
with head and breech presentations.

It is also known that ruptures often occur in the left half of the TC $[6,9,12,16]$. In our material tears TC in fetuses and newborns, born in the cephalic presentation, in most cases (69.2\%) are located in its left half TC and 30.8\% in the right half of TC. However, the reason for this is unclear. Only now, in view of our public laws it has become clear. Since the first position (fetus is turned back to the left side of the mother) occurs almost in 2 times more often than the second position, and the anterior asynclitism (presenting right parietal bone) more common posterior asynclitism, - in this situation the APS will more often displaced to the right parietal bone Figure 2. Consequently, in view of the said laws should expect more frequent tears in the left side of TC Figure 3, which occurs in reality.

Asynclitism - a deviation (offset) of the sagittal suture of the fetal head from the central axis of the birth canal of the mother. When there asynclitism, one parietal bone is at a lower level than the other. When there synclitism, two parietal bones are at the same level. At the anterior asynclitism sagittal suture moves backwards from the wire axis of the pelvis, towards the promontory of the sacrum - the parietal anterior insertion of the head. In this situation the anterior parietal bone (often right) is lower and the sagittal suture is near to the promontory. At the posterior asynclitism sagittal suture is displaced anterior to the wire axis of the pelvis towards the symphysis pubis - the parietal anterior insertion of the head.

Among the practical obstetrician there is an opinion that asynclitism not dangerous, it may occur in normal labour. They recognize the danger only the extreme asynclitism, when there are difficulties with the promotion of the head in the birth canal mother. Is it so? Our studies show that any degree asynclitism can be dangerous for the fetus. Much depends on the degree of the configuration [12] of the head. When there is configuration with synclitic insertion of the fetal head, the tension force, going from the falx cerebri, distributed evenly into the two halves TC. When there is asynclitic insertion head, the tensioning force is transferred to one of the halves of the TC, which can tear at the same degree head configuration. If at the same degree head configuration was observed synclitic insertion, the tear TC may not occur. To prevent tears TC (birth trauma), you must know the degree of the configuration of the fetal head during labor, and the exact degree of asynclitism. Finger vaginal examination is the century before last.

We studied the APS from dead fetuses and newborns, being born in the cephalic presentation and spontaneous labor. We have developed a patent for definition of the degree asynclitism and localization of the wired point of the fetal head $[19,20]$. APS clearly indicates insertion of the head, where is wire point, and what degree of asynclitism in millimeters.

We believe that it is necessary to rethink the role asynclitism to the fetus, and a new approach to its valuation. Normally wired point deviation of the head to $1.5 \mathrm{~cm}$ from the sagittal suture and does not lead to tears TC, but deviations greater size significantly increase the risk of tears. Hardly on digital vaginal examination can accurately determine the degree of asynclitism. Keep in mind also that asynclitism leads to uneven tension bridging veins, risking their breaks and the emergence of subdural and subarachnoid hemorrhage in the fetus. Asynclitism is not only obstetric problem is the problem of perinatal medicine in general.

\section{Results}

The results of morphological studies have shown that changes of the skull in newborns can exact location of the wired point of the head and measured in millimeters degree asynclitism. We have proved that any degree asynclitism unfavorable for the fetus, increasing the risk of birth trauma of the skull. Therefore it is necessary to review the concept of "Physiological Asynclitism".

Starting from the concept of the pathogenesis of TC tears becomes clear why at the asynclitic inserted head can not be used forceps and vacuum extractor. This will lead to increased tension of uneven TC and will dramatically increase risk of its tears.

In view of the findings pathologists and forensic experts are on morphological data, and not just the birth stories, to evaluate the quality of delivery in cases of death of children. Pediatric neurologists and neurosurgeons may use the data in the topical diagnosis of intracranial lesions. Obstetricians will be able to evaluate a new role asynclitic insertion of the head in the occurrence of intracranial lesions, because any degree asynclitism [12] accompanied by uneven tension of the two halves of the TC with an increased risk of damage to one of the parties. Asynclitic insertion of the head is favorable for the course of labor and promoting head through the birth canal, but unfavorable for the fetus in terms of increased risk of unilateral rupture TC. It is to prevent or reduce asynclitism will lead to a decrease in birth trauma.

Believe in the medicine of the future in all cases of births under medical supervision will be carried out dynamic monitoring of the progress of the head through the birth canal of a mother with a precise localization of wire point, the degree of asyclitism and nature of the insertion head. This is possible when using radial diagnostic methods in the process of childbirth. In medicine of the future will be established the exact diagnosis of damages TC (marker of birth trauma) both in labor and in newborns. Currently TC not examined, and the diagnosis of birth trauma is often difficult. Efforts in these areas will improve the prevention, diagnosis and treatment of birth trauma, and also will reduce mortality and reduce invalidism of children.

\section{Conclusion}

Diagnosis is important for the study of the APS:

- Evaluation of the insertion head,

- Determine the degree asynclitism,

- Localize wired point of head,

- $\quad$ Predict localization tears TC and

- $\quad$ Prognosis localization of subdural hemorrhage.

Any degree asynclitism leads to uneven tension TC and the 
risk of damage increases.

Asynclitic insertion of the head is a contraindication to the use of forceps and vacuum extractors.

Asynclitic insertion of the head leads to unilateral or predominantly unilateral tears of the TC.

\section{References}

1. Joseph J. Volpe. Neurology of the newborn. 5 Ed. Boston: Philadelphia: Elsevier Health Sciences; 2008.

2. John M. Optiz. Potter`s pathology of the fetus, infant and child. 2 nd Ed. Philadelphia: Mosby Elsevier; 2007.

3. Essbach H. Paidopathologie. Leipzig; 1961.

4. Tortori-Donati P, Rossi A., Raybaud C, Bianchery R. Pediatric Neuroradiology Brain. 1 Ed. New York: Springer-Verlag Berlin Heidelberg, 2005.

5. Stevenson DK, Benitz WE, Philip S. Fetal and Neonatal Brain Injury. 3 Ed. United Kingdom: Cambridge University Press, 2003.

6. Gurevich P.S. Sorokin A.F. Perinatal pathology. In: Ivanovskaya T.E. Leonova L.V., editors. Pathology of diseases of the fetus and child. V. 1. Moscow: Medicina; 1989. p. 38-121.

7. Beneke R. Uber Tentoriumzerreissungen bei der Geburt. Verh. Dtsch. Path. Ges. 14. Tagung. Erlangen. 1910: 46-54.

8. Coutelle $\mathrm{C}$. Inspection and tests of the mechanical strength of the Tentorium cerebelli in newborns and infants. Virchows Arch Pathol Anat Physiol Klin Med. 1960;333(1):10-21.

9. Astrinsky S., A. Kastner, Melenevskaya Z. On the question of injury to the cerebellar tentorium in the fetus during labor. Journal for the Study of Early Childhood. 1931; 12 (2): 93-101.

10. Schwartz P. Birth injuries of the newborn. Jena: Springer Verlag, 1964.

11. Kastner A.G. Birth injury to the fetus. In: Davydovsky I.V. Pathological anatomy and pathogenesis of the human disease. Moscow: Medicina; 1958. p. 528-549.

12. Vlasyuk V.V. Birth trauma and perinatal disorders of cerebral circulation. St.Petersburg: Nestor-Istoriya Publ.; 2009.

13. Vlasyuk V.V. Brain pathology in infants and young children. Moscow: Logosphera, 2014.

14. Vlasyuk V.V. On the causes and mechanisms of subdural hemorrhages and ruptures of cerebellar tentorium in fetuses and newborns. Pediatriya. 1985; 9: 6-9.

15. Vlasyuk V.V., Lobzin Yu.V., Nesmeyanov A.A. Postmortem assessment of labor from changes in the skull and brain in fetuses and newborn infants. Arkhiv Patalogii. 2014;76(3): 74-79.

16. Popova NI. Morphological characterization of intracranial hemorrhage due to birth trauma. Issues of maternal and child health. 1968;1:1925.

17. Mironchik I.N. Perinatal autopsy. Minsk, 1981.

18. Krasovsky E.B., Glagoleva M.A. About mortem diagnosis of birth injury. In: Perinatal Pathology and Teratology. Saratov, 1980, p. 26-27.

19. Vlasyuk V.V., Lobzin Yu.V., Nesmeyanov A.A. The method of diagnosing asynclitism according to the skull changes. Patent RF № 2422087, 18.02.2010

20.Vlasyuk V.V., Lobzin Yu.V., Nesmeyanov A.A. The method of determining localization the wired point of the head according to the skull changes in the deceased fetuses and newborns. Patent RF № $2470583,27.12 .2012$. 